
\title{
Research S Surare \\ The Expected Length of Stay At Aged Care Facilities in Australia: Current and Future
}

\author{
Yanlin Shi \\ Macquarie University \\ Jinhui Zhang ( $\nabla$ colin.zhang@mq.edu.au ) \\ Macquarie University
}

\section{Research Article}

Keywords: heterogeneous, dataset, covariate

Posted Date: August 30th, 2021

DOl: https://doi.org/10.21203/rs.3.rs-800309/v1

License: (9) This work is licensed under a Creative Commons Attribution 4.0 International License. Read Full License 


\title{
The expected length of stay at aged care facilities in Australia: Current and future
}

\author{
Yanlin Shi \\ Department of Actuarial Studies and \\ Business Analytics, Macquarie University, \\ Sydney, NSW, Australia, 2109.
}

\author{
Jinhui Zhang \\ Department of Actuarial Studies and \\ Business Analytics, Macquarie University, \\ Sydney, NSW, Australia, 2109. \\ Corresponding author. \\ Email: colin.zhang@mq.edu.au \\ Tel: +61298508484
}

\begin{abstract}
This paper analyzes the hazard functions of exiting from an aged care facility in Australia. Using a comprehensive dataset ranging over 2008-2018, we find that those functions are heterogeneous across the age, sex and yearof-leaving. The modelling results lead to in-sample estimated expected length of stay (LOS) for residents differed by age (in general, longer for older groups) and sex (longer for females). The estimated LOS declines gradually from 2008 to 2014 and then steadily increase afterwards for all heterogeneous age and sex groups. Out-of-sample predictions up to 2100 suggest that the longest LOS belongs to females aged 100 and older, with the estimated/predicted LOS increasing from 58.6 months in 2018 to 68.9 months in 2100 . Relative uncertainty measures are also provided. Those results can be used to explore the nature of and aspects to improve service quality of Australian aged care facilities for policy makers.
\end{abstract}




\section{Introduction}

According to [1], the life expectancy in Australia has been significantly rising for both male and female over the last century. Specifically, the life expectancy at birth is 80.4 for male and 84.6 for female, as of 2020. The Australian government is currently funding $\$ 18$ billion per year to the aged care service sector, where the majority of the government fund $(67 \%)$ has been used for residential aged care sector [2]. Based on the current demographic projections, the population number of senior Australian who are aged 85 or above will rise from 500,000 to 1,000,000 by 2042 [3]. The statistics from [4] show that more than $20 \%$ of the people aged 85 and above would like to use the residential aged care service. Hence, the Australian government may face a heavier budget burden in the future to provide the growing aging population with financial supports for the aged care service [5].

The prominence of population ageing can deeply impact people's financial behaviour. Based on the means-test, aged care users can receive different levels of financial supports from the government. According to [6], senior Australian aged care residents pay around $25 \%$ of the total aged care cost, while the remaining amount is funded by the government. In general, aged care residents can be classified as fully supported, partially supported and self-funded residents. The partially supported and self-funded residents need to make personal contributions for the service. Therefore, the public has an increasing interest in understanding the hazard functions to leave and the length of stay (LOS) at an aged care facility. This may help them make optimal financial decisions regarding their future plans.

Currently, residential aged care facilities are used by a large number of vulnerable senior citizens for late-life care [7]. Compared to the general population, people who live in the aged care facilities have higher mortality rate $[8,9]$. The high mortality and other exit rates indicate that there is a demand to include the palliative care into the aged care facilities. This needs to be met and addressed in relevant future policies.

In this paper, we study the hazard functions to exit from a residential aged care facility, focusing on the palliative care data. The dataset is sourced from the Australian Institute of Health and Welfare (AIHW), spanning from 2008 to 2018. We firstly employ the weighted Cox regression developed in [10] to preliminarily determine the significant factors that influence the hazard ratios to leave an aged care. The benefit of this method is that the validity of parameter inference is not affected by the violation of the proportional assumption, a critical feature of the ordinary Cox regression [11]. Further, using the identified influential factors, the age, sex and 
year-of-leaving, we employ the Cox regression to study in-sample and predict the out-of-sample LOS up to 2100. The violated proportional assumption is addressed via the stratification on age and sex and application of the restricted cubic splines (RCS) for the logged year-of-leaving. Our results provide both in-sample estimated and out-of-sample predicted expected LOS for aged care residents of heterogeneous groups. This can be used to investigate the nature of and build a foundation for quality improvement of aged care facilities for policy makers.

\section{Methods}

\section{$2.1 \quad$ Model}

To estimate the expected LOS at an aged care facility, regression-based models may be employed. However, the exact length is not available for all aged care residents. This is due to reasons of exiting, such as transferring to other aged care facilities. See Section 3.1 for more details. This incomplete observation is therefore a right-censored case in the survival analysis, and we employ the popular Cox regression [11] specified below in this paper:

$$
\begin{aligned}
h_{i}(t) & =h_{0}(t) e^{\eta_{i}} \\
\eta_{i} & =\beta_{1} x_{i, 1}+\beta_{2} x_{i, 2}+\ldots+\beta_{k} x_{i, k}
\end{aligned}
$$

where $h_{i}(t)$ is the hazard ratio to exit from an aged care of the $i$ th observation at time $t, h_{0}(t)$ is the unspecified baseline hazard ratio at time $t$, and $x$ 's are the corresponding covariates that will influence the LOS at an aged care via $h_{i}(t)$.

An essential assumption for a valid Cox regression is the proportional hazards. For two observations $i$ and $j$, using (2.1), it can be shown that

$$
\frac{h_{i}(t)}{h_{j}(t)}=\frac{h_{0}(t) e^{\eta_{i}}}{h_{0}(t) e^{\eta_{j}}}=\frac{e^{\eta_{i}}}{e^{\eta_{j}}} .
$$

In other words, at the same time $t$, hazard ratios of two observations are independent of $t$. Intuitively, this means that the corresponding survival curves will not cross for any two observations over the entire sample period.

If this assumption is violated, non-proportionality will often lead to inaccurate results. For instance, estimates under non-proportionality are argued to be sensitive to the type of departure from proportionality and censoring pattern of the data [12, 13]. If the covariate is categorical, a simple solution is to adopt the stratification. 
However, the significance of effect of the stratifying covariate cannot be examined in this case [10].

To resolve this issue, many extensions of the Cox regression have been proposed. Early attempts can be found in [14] and [15], among others. More recently, [12] propose a weighted Cox regression. The advantage (disadvantage) of this method is that it provides an (uninterpretable) average effect that is independent of the observed censoring pattern. [10] further extend the weighted approach with a revised weighting strategy, such that the estimated average effect could be interpretable. Moreover, this improved weighted Cox regression is more efficient than the concordance regression [13], which is also independent from type of nonproportionalhazards and censoring pattern. However, a significant drawback of this weighted Cox regression is that a prediction cannot be performed.

In this paper, we therefore employ the weighted Cox regression studied in [10] to investigate significance of covariates, for its improved efficiency and interpretability. A standard Cox regression is then fitted with selected covariates for the prediction purpose. Specifically, in a weighted Cox regression, estimates of coefficients in (2.1) are obtained by solving the following set of equations

$$
\frac{\partial \log L(\boldsymbol{\beta})}{\partial \beta_{r}}=\sum_{j=1}^{m} w\left(t_{j}\right)\left[x_{j, r}-\frac{\sum_{l \in R_{j}} x_{l, r} e^{\eta_{l}}}{\sum_{l \in R_{j}} e^{\eta_{l}}}\right]
$$

where $\log L(\boldsymbol{\beta})$ is the $\log$ likelihood function corresponding to $(2.1), \boldsymbol{\beta}=$ $\left(\beta_{1}, \beta_{2}, \ldots, \beta_{k}\right)^{\prime}, t_{j}$ is the $j$ th distinct uncensored survival times, $m$ is the total number of those distinct times, $R_{j}$ is the set of subjects without the event and uncensored prior to $t_{j}$ (also known as the risk set), and $w\left(t_{j}\right)$ is the weight. If all $w\left(t_{j}\right)$ are set to 1 , then a standard Cox regression is solved. Let $S(t)$ the survival function at time $t$ and $G(t)$ the cumulative probability of follow-up until $t,[10]$ require that $w\left(t_{j}\right)=S\left(t_{j}\right) G\left(t_{j}\right)^{-1}$. Intuitively, those weights are proportional to the expected number of subjects at risk at each $t_{j}$ if there had been no censoring. This then improve the interpretability of estimated coefficients, compared to those with weights set to $G\left(t_{j}\right)^{-1}$ as in $[12]$.

After a weighted Cox regression is fitted, a standard Cox model is then constructed to obtain predictions, using selected influential covariates in the weighted model. In this standard regression, a categorical covariate will be stratified, if the corresponding nonproportional-hazards assumption is violated. Under the more complicated scenario such that non-proportionality is found for a non-categorical variable, we consider including time-by-covariate interactions. Specifically, as recommend by [16], the restricted cubic splines is employed in such cases, to allow for 
a parsimonious but flexible estimation of time-dependent effects for non-categorical covariates.

\section{Data and Results}

\subsection{Data}

We use the palliative aged care data sourced from the Australian Institute of Health and Welfare (AIHW) (https://www.gen-agedcaredata.gov.au/ Resources/Access-data/2020/August/GEN-data-People-leaving-aged-care) for this research. Since this dataset is the existing publicly non-identifiable accessible record, this research is exempted from ethical review. This dataset contains around 750,000 records of residents leaving their aged care facilities during the period from 2008 to 2018. Detailed information of residents, which includes year-of-leaving, state of aged care facility, discharge reason, LOS, age group, gender, indigenous status, preferred language and country of birth, is recorded in this dataset.

To facilitate our research, a censorship takes place if an aged care resident is transferred to other aged care facilities, or exits without a specified reason. Also, to be consistent with existing studies such as [7], we employ the LOS measured in months. The dataset is further refined such that there are no missing data in each of the following (categorical) covariates of an aged care resident: sex, age, residential state, indigenous status, country of birth and language spoken. Section 3.1.1 describes the investigated sample across those covariates with all year-of-leaving (2008-2018) combined. In section 3.1.2, we present the temporal trends and compare relevant differences.

\subsubsection{All year-of-leaving combined}

We firstly consider the one-dimensional distribution according to each of the examined categorical covariates, with data spanning 2008-2018 combined. Altogether, there are 737,750 observations in our dataset. The distributions (measured in percentages) are listed in Table 1 . Overall, it can be seen that female residents are dominant (around 60\%), and over one fourth are 85-89 years old. As for the residential state, New South Wales (NSW) and Victoria (VIC) take up over 60\%. Further, the non-indigenous population is the majority (99.3\%), most aged care residents were born in Australia and other English-speaking countries (nearly 85\%), and over 90\% 
of all residents can speak English. Many of the above distributions (except those of sex and age) are much consistent with their counterparts for the general Australian population. See [17] and [18] for details of such distributions.

Table 1: Distributions of categorical covariates, measured in percentages

\begin{tabular}{|c|c|c|c|c|c|c|c|c|c|c|c|}
\hline \multicolumn{2}{|c|}{ Sex } & \multicolumn{2}{|c|}{ Age } & \multicolumn{2}{|c|}{ State } & \multicolumn{2}{|c|}{ Indigenous } & \multicolumn{2}{|c|}{ Country of Birth } & \multicolumn{2}{|l|}{ Language } \\
\hline $\mathrm{F}$ & 61.7 & $0-49$ & 0.3 & NSW & 35.2 & Yes & 0.7 & Australia & 71.1 & English & 91.0 \\
\hline M & 38.3 & $50-54$ & 0.3 & VIC & 25.7 & No & 99.3 & Non-English & 16.4 & Other languages & 9.0 \\
\hline & & $55-59$ & 0.7 & QLD & 17.7 & & & Other English & 12.5 & & \\
\hline & & $60-64$ & 1.3 & SA & 8.9 & & & & & & \\
\hline & & $65-69$ & 2.6 & WA & 8.3 & & & & & & \\
\hline & & $70-74$ & 4.9 & TAS & 2.8 & & & & & & \\
\hline & & $75-79$ & 9.4 & $\mathrm{ACT}$ & 1.1 & & & & & & \\
\hline & & $80-84$ & 18.1 & NT & 0.2 & & & & & & \\
\hline & & $85-89$ & 27.0 & & & & & & & & \\
\hline & & $90-94$ & 23.5 & & & & & & & & \\
\hline & & 95-99 & 9.9 & & & & & & & & \\
\hline & & $100+$ & 1.9 & & & & & & & & \\
\hline
\end{tabular}

Using the uncensored (complete) data only, we now discuss the average LOS at an aged care. There are 616,136 such observations in our dataset, indicating a censoring ratio of $16.5 \%$. Note that the average lengths of the full, censored and uncensored samples are 28.3, 24.4 and 29.1, respectively. Average lengths according to each of the categorical variates are presented in Table 2. We observe that females (33.3 months) spend $50 \%$ more time on average than males (22.4 months) at an aged care. Also, the average LOS for ages groups within 0-84 are close to each other, and are mostly around 23 months. On the residential state dimension, only the average length of Northern Territory (32.5 months), or NT, deviates more than trivially from the rest (around 29 months). Non-indigenous residents only spend few more months at an aged care, on average, than indigenous users. There are also not much differences at the subcategory level, when country of birth and language spoken are individually considered.

Table 2: Average LOS in months at an aged care (uncensored data)

\begin{tabular}{|c|c|c|c|c|c|c|c|c|c|c|c|}
\hline \multicolumn{2}{|c|}{ Sex } & \multicolumn{2}{|c|}{ Age } & \multicolumn{2}{|c|}{ State } & \multicolumn{2}{|c|}{ Indigenous } & \multicolumn{2}{|c|}{ Country of Birth } & \multicolumn{2}{|l|}{ Language } \\
\hline $\mathrm{F}$ & 33.3 & $0-49$ & 24.1 & NSW & 28.0 & Yes & 27.6 & Australia & 29.6 & English & 29.0 \\
\hline \multirow[t]{11}{*}{$\mathrm{M}$} & 22.4 & $50-54$ & 21.9 & VIC & 29.2 & No & 29.1 & Non-English & 28.1 & Other languages & 30.0 \\
\hline & & $55-59$ & 22.8 & QLD & 29.9 & & & Other English & 27.7 & & \\
\hline & & $60-64$ & 23.2 & SA & 31.1 & & & & & & \\
\hline & & $65-69$ & 23.1 & WA & 29.4 & & & & & & \\
\hline & & $70-74$ & 23.0 & TAS & 28.8 & & & & & & \\
\hline & & $75-79$ & 23.3 & $\mathrm{ACT}$ & 29.0 & & & & & & \\
\hline & & $80-84$ & 24.3 & NT & 32.5 & & & & & & \\
\hline & & $85-89$ & 27.1 & & & & & & & & \\
\hline & & $90-94$ & 32.4 & & & & & & & & \\
\hline & & $95-99$ & 39.9 & & & & & & & & \\
\hline & & $100+$ & 52.2 & & & & & & & & \\
\hline
\end{tabular}

Some differences observed in Table 2 may be explained by the age-specific distributions at subcategory level of each covariate. To demonstrate this, we plot those 
distributions in Figure 1 (b)-(f). For instance, there are relatively more older residents in states other than NT. More discussions regarding this are provided in Section 3.1.3, and formal tests are conducted in Section 3.2.

\subsubsection{Temporal patterns}

We now present the potential temporal patterns in the average LOS at an aged care, by considering the yearly data separately. In Figure 2 (a), it can be seen that those averages almost monotonically grow with time, from 26.8 in 2008 to 31.2 in 2018.

Temporal patterns are further investigated for each of the categorical covariates, in Figure 2 (b)-(g). As for the sex, despite the growing trends, females spend consistently more time than males in all years. Their differences in each individual year are much close to each other and at around 11 months. Regarding the age groups, "parallel" curves are observed for the four oldest age groups (85-89, 90-94, 95-99 and 100+). Much more volatile patterns exist for younger groups (e.g., 0-49), which may be explained by the small sample size, as indicated in Table 1. Temporal patterns across states are also volatile, especially for those with small populations, including Australian Capital Territory (ACT), Tasmania (TAS) and NT. Despite the volatility, roughly speaking, only residents in ACT and TAS do not spend more time at an aged care over years. Moreover, for all states except NT, the differences among their average LOS in each year are considerably small. Due to the small population (250-550 in each year), average LOS are too volatile to draw a temporal trend for indigenous aged care residents. As for the country of birth, residents born in Australia spend more time at an aged care than those born elsewhere. The differences, however, are reducing quickly over years. Finally, there seems to be a steeper slope for the temporal trend of non-English-speaking aged care users than English-speaking residents. Despite this, the largest difference among all years is only 2 months.

Overall, it appears that there may exist significant temporal trends at the subcategory level of all relevant covariates. Recall that there are potentially significant differences in average LOS across (old) age groups. Thus, temporal changes in agespecific distribution of a covariate may explain the corresponding yearly patterns in the average LOS. For instance, it can be seen in Figure 1 (a) that the curve gradually shifts to the right side (older ages) over time. Thus, even if there is no temporal changes for the underlying age-specific hazard ratio to exit an aged care, with more older residents over years, a temporal trend similar to Figure 2 (a) will be observed. We therefore discuss temporal changes of age and sex-specific distributions of other 
covariates in next section.

\subsubsection{Age and sex-specific pyramids}

Based on the empirical evidence, it is credible to consider that age and sex are influential covariates on the mortality [19] and therefore on hazard ratios of aged care LOS. Consequently, we examine dynamic changes in age and sex-specific distributions for the investigated covariates. Those may help explain the observed differences of some covariates in Section 3.1.2. Specifically, following [18], demographic pyramids are presented, which are useful to demonstrate age and sex-specific distributions simultaneously. Also, to further incorporate the temporal changes, we contrast distributions of 2008 and 2018 in those pyramids.

First, pyramids are produced for each state in Figure 3. Consistent in all states except NT, there are more older female than male aged care residents. Also, for both sexes, proportions of older ages are growing with time. For instance, in all states except NT, the dominant age group in females changes from 85-89 in 2008 to 90-94 in 2018. Due to the small population of NT residents, the distributions are more irregular and differ from those of other states. This may explain the differences among temporal trends presents in Figure 2 (d).

Additionally, pyramids at subcategory level are generated for indigenous status, country of birth and languages spoken in Figures 4, 5 and 6, respectively. It seems that the changes in age and sex-specific distributions of the indigenous status may help explain the observed differences in Table 2 and Figure 2 (e), but not for other covariates. Formal tests are therefore conducted in Section 3.2.

\subsection{Preliminary analyses}

We now consider preliminary pairwise analyses to illustrate potential influence of each categorical covariate. In all cases, the subsample of 2008 is employed for demonstrative purposes. Also, the traditional log-rank test is not employed to compare whether two fitted survival curves differ significantly. The reason is that its power will reduce when the hazard ratio is not constant, especially when those ratios of two curves cross [20-22]. Instead, the adaptively weighed log-rank (AWL) test proposed in [23] is employed, which is more robust when the hazard ratio is not constant.

First, we focus on the covariate of age, using the largest age group 85-89 and the next younger category (80-84) for comparison. To control for the potential interac- 
tion between sex and age, the subgroup of females is analyzed. The corresponding fitted Kaplan-Meier (KM) survival curves with 95\% confidence intervals (CIs) are plotted in Figure 7 (a). Significant differences are observed at least over months $(0,50)$. The p-value of AWL test is 0 , suggesting that age is a significant factor influencing the hazard ratio.

Next, we consider the variable of sex. For the same reason of possible interactions, the largest age group 85-89 is examined. As shown in Figure 7 (b), over months $(0,150)$, females are more likely than males to stay at an aged care. The p-value of AWL test is 0 , indicating that sex is also an important covariate.

Since age and sex are both potentially influential, we use female that were aged 85-89 and left the facility in 2008 in the following analyses. To illustrate the impact of state and country of birth, the pair of ACT versus NT and Australia versus nonEnglish-speaking countries are compared, respectively. The binary subcategories in indigenous status and language spoken are also contrasted. The fitted KM survival curves are plotted in Figure 7 (c)-(f). Using the AWL test, the only covariate that is significantly influential on the hazard ratio of aged care stay is the language spoken.

Weighted Cox regression models are now fitted to provide more informative results on the significance of categorical covariates. The main model is the average hazard ratios (AHR) proposed in [10], and the average regression effects (ARE) developed in $[12]$ is employed as the robustness check.

We firstly focus on the subsample of 2008 with age of 85-89 to illustrate the significance of sex, residential state, indigenous status, language spoken and country of birth. Without loss of generality, the baseline subcategories are chosen alphabetically as female, ACT, indigenous, English and Australia, respectively. Results are presented in the left panel of Table 3. It can be seen that the only significant (at 1\%) covariate is sex, and the factor of language spoken is at the boundary level ( $\mathrm{p}$-value of 0.047) according to AHR, and insignificant (p-value of 0.120) based on ARE. Second, we analyze the impact of the non-categorical variable of year-of-leaving. Using female data aged 85-89, we fit this single covariate using weighted Cox regression with AHR and ARE. The results are reported in the middle panel of Table 3. The logged year-of-leaving variable is therefore significant in affecting the underlying hazard ratio. Thus, at this stage, our preliminary analyses suggest that covariates of sex and year-of-leaving are influential to study the aged care LOS.

Recall in Section 2.1, weighted Cox regression cannot be employed for the prediction purpose. Instead, the standard Cox model needs to be used. In such a case, if the non-proportional assumption is violated, the categorical variables need to be stratified. However, this is only practical for covariates with a small number 
Table 3: Preliminary analyses: Weighted Cox regression

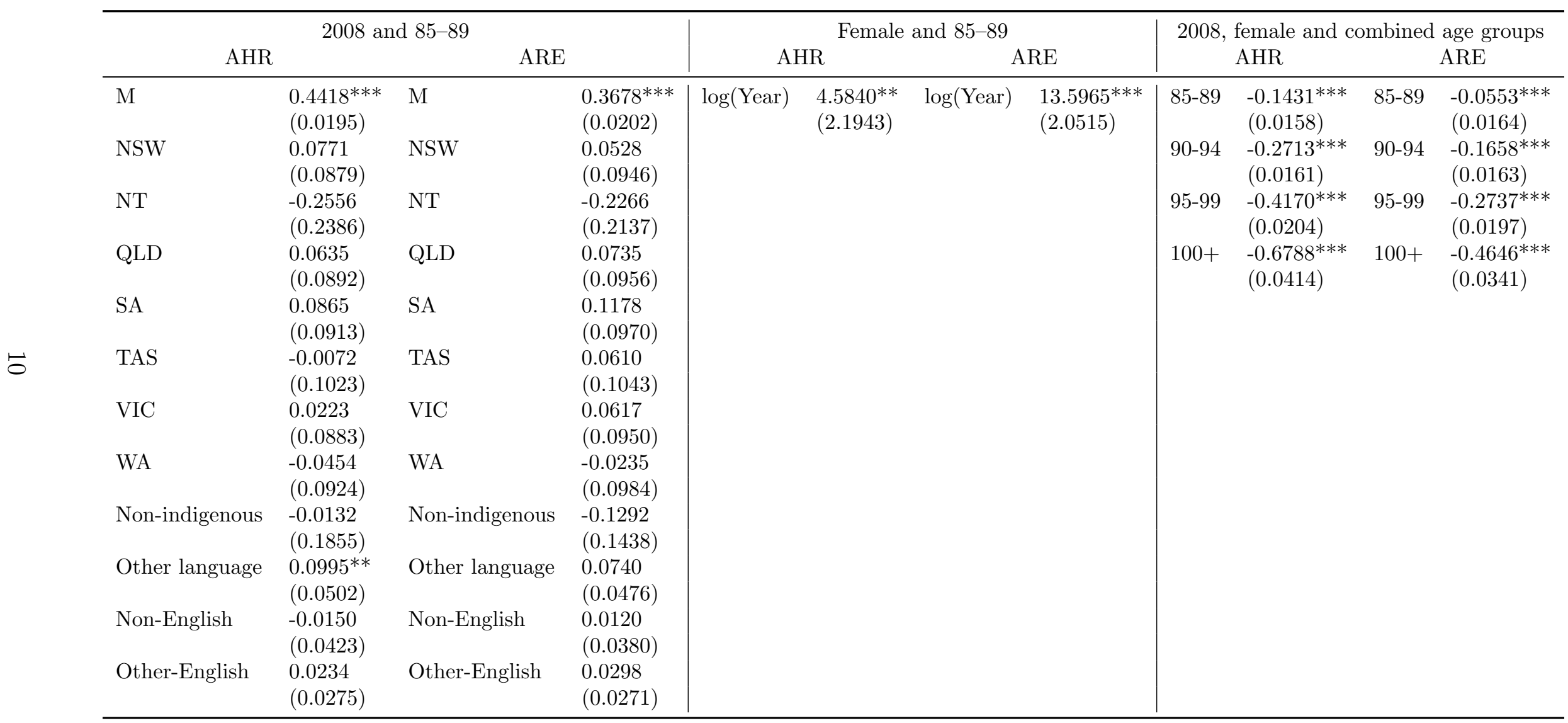

Note: AHR and ARE are weighted Cox regressions with average hazard ratios [10] and average regression effects [12], respectively. Values in parentheses are the corresponding robust standard errors [24]. ${ }^{*}, * *$ and $* * *$ indicate the significance at $10 \%, 5 \%$ and $1 \%$ level, respectively. 
of subcategories [16]. Further, as demonstrated in Table 2, the average lengths of aged care stay for younger age groups may not differ largely from each other. Thus, it is worth investigating if some subcategories of the age could be combined, before we formally test its significance on influencing the aged care LOS.

For this aim, we employ the uncensored data only, and display the age and sexspecific average LOS in Figure 8. Specifically, the female and male yearly averages are presented in (a) and (c), respectively, whereas the female and male means considering all years are plotted in (b) and (d), respectively. For the yearly cases, much volatility is observed for younger ages (especially for males younger than 70), which may be explained by their small population sizes. Using the combined plots, it can be seen that the average lengths of females (males) younger than 85 (90) are almost identical. This then provides motivation to combine age groups younger than 85 as a new group, named 0-84. Figure 9 therefore illustrates average lengths using this new subcategory. It can be seen that the yearly curves are more stable with much distinct differences from one group to another. Despite that the average lengths for males aged 0-84 and 85-89 are similar, this new division is more general and helps distinguish the differences in female age groups.

Weighted Cox regressions are then fitted to preliminarily demonstrate the significant differences among age groups. Using the female data of 2008, the results are presented in the right panel of Table 3. The baseline case is the new 0-84 group. Clearly, older subcategories are individually significant at $1 \%$ in both AHR and ARE models.

In summary, our preliminary analyses suggest that the three influential covariates on the hazard ratio of aged care LOS are sex, age and year-of-leaving. Specifically, significant estimates in Table 3 indicate that with all other covariates held the same, males are more likely to exit an aged care compared to females; older residents are expected to stay longer; and within our sample period, residents may stay shorter over time (considering a linear trend only in the logged year-of-leaving). We discuss the final model using those covariates in a standard Cox model and the predicted results in next section.

\subsection{The final model and predictions}

A standard Cox model consisting of logged year-of-leaving, age (with five subgroups: 0-84, 85-89, 90-94, 95-99 and 100+) and sex is firstly examined. To investigate if the proportional-hazards assumption is met, we perform the test proposed in [25]. The $\chi^{2}$ test statistics of logged year-of-leaving, age and sex are 675,7653 
and 3163, respectively, indicating significant non-proportional hazards in all cases. As stated in Section 2.1 and recommended by [16], we then apply stratification on age and sex, and employ the restricted cubic splines (RCS) for the logged yearof-leaving. To select the degrees of freedom (knots) of RCS, we use the Bayesian information criterion, which favors more parsimonious specifications.

The final stand Cox model is composed of RCS of logged year-of-leaving with 3 degrees of freedom, as well as stratified age and sex. Both the log-likelihood ratio and score tests suggest that RCS of logged year-of-leaving can significantly influence the hazard ratio of aged care stay, after considering stratified age and sex. The test statistics are 65.58 and 65.32 , respectively, and both follow a $\chi^{2}$ distribution with 2 degrees of freedom. We therefore use the fitted coefficients to estimate in-sample and predict out-of-sample hazard ratios. The corresponding survival curves and expected aged care LOS can then be produced.

We firstly compare the in-sample estimates of expected aged care LOS using the final model to those fitted by the KM method. Results are plotted in Figure 10. Note that the KM estimates are obtained using subsample that considers the three covariates jointly. For instance, the 2008 female aged 0-84 sample is employed to produce the corresponding KM result. In contrast, the Cox regression utilizes the full sample (2008-2018) in all cases. This may explain the relative stability of its estimates over the KM counterparts. For instance, the KM results for aged $100+$ are more volatile over years, due to the small subsample sizes. Despite this, the Cox results are much close to the KM estimates, which are used as the benchmark (true values). It is worth noting that after considering age, sex and their interaction, the expected LOS actually gradually declines over years 2008-2014 in most cases. This is different from preliminary observations presented in Section 3.1.

The above results suggest that the fitted Cox model is able to provide accurate in-sample estimates. Since the RCS of logged year-of-leaving is parsimonious, the overfitting issue is much avoided, and the out-of-sample prediction is therefore expected to be reliable. The fitted and predicted expected aged care LOS over 20082100 with 95\% CIs using the final model are plotted in Figure 11. In all cases, the results gradually grow over time, after a short declining trend spanning from 2008 to 2014. For instance, the final model suggests that the expected length of aged care stay for female aged 0-84 reduces from 35.4 months in 2008 to 34.0 months in 2014 . It then stably increases from 34.1 months in 2015 to 43.6 months in 2100. Contrasting estimates/predictions of 2018 and 2100, on average, aged care LOS increases by 9.3, 8.1, 8.4, 9.2 and 10.3 months, respectively, for females aged 0-84, 85-89, 90-94, 95-99 and 100+. The male counterparts are relatively shorter and are 8.4, 6.7, 6.7, 
7.5 and 8.5 months, respectively. As of 2018, the shortest expected aged care LOS is 24.9 months for male aged $85-89$, and the longest is 58.6 months for female aged $100+$. In 2100, the shortest and longest expected lengths are 31.7 months for male aged 85-89, and 68.9 months for female aged $100+$, respectively.

Finally, we investigate the fitted and predicted survival curves of aged care stay in 2008 and 2100, respectively. The curves (with 95\% CIs) are plotted in Figure 12. For the same age and sex-specific subcategory, the predicted curve shifts to the right from 2008 to 2100, indicating a longer expected LOS. Additionally, the uncertainty grows substantially, comparing the widths of CIs in 2008 and 2100. In particular, the survival curves of male and female aged 100+ are not significantly different from each other, from the 75th month onwards, as of 2100.

\subsection{Discussions}

From our preliminary analyses and final model, we identify that sex, age and year-of-leaving are three impactive factors on the hazard ratios to leave from an Australian aged care facility. The significant influence of sex is well consistent with existing studies and statistics. For instance, empirical evidence shows that female generally has lower mortality rates and longer life expectancy than males [3].

Our preliminary results indicate that in general, older residents are expected to stay longer at an aged care. This is also consistent with existing evidence. Statistics show that the demand of aged care usually increases with age [26], and the majority of residential aged care residents are people aged 85 and above [27]. In comparison, younger aged care residents usually require high-level care [28] and have higher mortality rates caused by external factors [29]. This may explain their estimated higher exit rates in our empirical results.

Our empirical results also suggest that the aged care LOS decreases along the yearof-leaving between 2008 and 2014. This may be partially explained by the decrease in admission to residential aged care facilities, which concurs with the appearance of home care packages service, during this period [30].

In Section 3.3, we use the fitted Cox model to predict the future aged care LOS for heterogeneous age and sex groups. Longer LOS is evidenced in all cases. Based on our results, the oldest age group $(100+)$ of both gender have the largest increase in predicted LOS. Thus, with the evidenced increasing of Australian life expectancies [3], policy makers need to consider the special needs of centenarians residents in an aged care facility. Those residents and their family members will have to account for such increasing expenditures in their future personal financial plans. 


\section{Concluding remarks}

In this paper, we work on the palliative data over 2008-2018 sourced from the Australian Institute of Health and Welfare. Preliminary analyses are firstly performed to identify the key covariates which profoundly impact the hazard ratios of aged care stay. Our analyses indicate that sex, age and year-of-leaving are three prominent covariates which should be included in our final prediction model.

Using a Cox regression with stratified age and sex and RCS applied to the logged year-of-leaving, we estimate the in-sample (2008-2018) and predict out-of-sample (2019-2100) LOS and associated survival curves. Our results demonstrate longer terms of aged care LOS over the prediction period. Specifically, the older age groups (e.g., 95-99 and 100+) are expected to have larger grows in the expected LOS. Meanwhile, our model can also facilitate the uncertainty measures in the future survival curves of aged care stay. Overall, our results point out the importance of integrating the palliative care services into the residential aged care.

An aging population with longer aged care LOS will be likely associated with a rising aged care cost in the future. We hope this paper can trigger the awareness of this issue for both individual and policymakers to make relevant personal and public plans.

\section{Author Contributions}

J.Z. designed the research. Y.S. performed the research and analyzed the data. Y.S and J.Z. wrote the paper.

\section{Competing interests}

This research did not receive any specific grant from funding agencies in the public, commercial, or not-for-profit sectors. The authors declare no competing interests.

\section{References}

1. Australia Institute of Health and Welfare. Deaths in Australia. Australia Government. https : //www . aihw . gov . au/reports/life-expectancy-death/ deaths-in-australia/contents/summary (2020). 
2. Australia Institute of Health and Welfare. Government spending on aged care. Australia Government. https : / www . gen-agedcaredata .gov . au/Topics / Government-spending-on-aged-care (2019).

3. Australia Bureau of Statistics. Population Projections, Australia. Australia Government. https://www .abs.gov .au/statistics/people/population/ population - projections - australia / 2017 - base - 2066 \$ \\#articles (2018).

4. Australia Institute of Health and Welfare. Residential aged care in Australia 2010-11: A statistical overview. Australia Government. https://www . aihw . gov . au / reports / aged - care / residential - aged-care-in-australia2010-11/contents/table-of-contents (2012).

5. Ergas, H. \& Paolucci, F. Providing and financing aged care in Australia. Risk management and healthcare policy 4, 67 (2011).

6. Australian Aged Care Collaboration. Aged care-the way forward. https : // apo . org . au / sites / default / files / resource-files / 2021 - 04 / aponid311764.pdf (2021).

7. Broad, J. B. et al. Likelihood of residential aged care use in later life: a simple approach to estimation with international comparison. Australian and New Zealand journal of public health 39, 374-379 (2015).

8. Shah, S. M., Carey, I. M., Harris, T., DeWilde, S. \& Cook, D. G. Mortality in older care home residents in England and Wales. Age and ageing 42, 209-215 (2013).

9. Ferrah, N., Ibrahim, J. E., Kipsaina, C. \& Bugeja, L. Death following recent admission into nursing home from community living: a systematic review into the transition process. Journal of aging and health 30, 584-604 (2018).

10. Schemper, M., Wakounig, S. \& Heinze, G. The estimation of average hazard ratios by weighted Cox regression. Statistics in medicine 28, 2473-2489 (2009).

11. Cox, D. R. Regression models and life-tables. Journal of the Royal Statistical Society: Series B (Methodological) 34, 187-202 (1972).

12. $\mathrm{Xu}, \mathrm{R}$. \& O'Quigley, J. Estimating average regression effect under nonproportional hazards. Biostatistics 1, 423-439 (2000).

13. Dunkler, D., Schemper, M. \& Heinze, G. Gene selection in microarray survival studies under possibly non-proportional hazards. Bioinformatics 26, 784-790 (2010).

14. Schemper, M. Cox analysis of survival data with non-proportional hazard functions. Journal of the Royal Statistical Society: Series D (The Statistician) 41, 455-465 (1992).

15. Sasieni, P. Maximum weighted partial likelihood estimators for the Cox model. Journal of the American Statistical Association 88, 144-152 (1993).

16. Dunkler, D., Ploner, M., Schemper, M. \& Heinze, G. Weighted Cox Regression Using the R Package coxphw. Journal of Statistical Software 84, 1-26 (2018).

17. Raymer, J., Shi, Y., O'Donnell, J. \& Biddle, N. Multistate projections of Australia's Indigenous population. Vienna Yearbook of Population Research 16, 135-162 (2018). 
18. Raymer, J., Shi, Y., Guan, Q., Baffour, B. \& Wilson, T. The sources and diversity of immigrant population change in Australia, 1981-2011. Demography 55, 1777-1802 (2018).

19. Australia Bureau of Statistics. Deaths, Australia. Australia Government. https : / / www . abs . gov . au/statistics / people / population / deaths australia/latest-release (2019).

20. Yang, S. \& Prentice, R. Semiparametric analysis of short-term and long-term hazard ratios with two-sample survival data. Biometrika 92, 1-17 (2005).

21. Yang, S. \& Prentice, R. L. Estimation of the 2-sample hazard ratio function using a semiparametric model. Biostatistics 12, 354-368 (2011).

22. Yang, S. \& Zhao, Y. Checking the short-term and long-term hazard ratio model for survival data. Scandinavian Journal of statistics 39, 554-567 (2012).

23. Yang, S. \& Prentice, R. Improved logrank-type tests for survival data using adaptive weights. Biometrics 66, 30-38 (2010).

24. Lin, D. Y. \& Wei, L.-J. The robust inference for the Cox proportional hazards model. Journal of the American statistical Association 84, 1074-1078 (1989).

25. Grambsch, P. M. \& Therneau, T. M. Proportional hazards tests and diagnostics based on weighted residuals. Biometrika 81, 515-526 (1994).

26. Rahman, M., Efird, J. T. \& Byles, J. E. Patterns of aged care use among older Australian women: a prospective cohort study using linked data. Archives of gerontology and geriatrics 81, 39-47 (2019).

27. Australia Institute of Health and Welfare. People using aged care. Australia Government. https : / / www . gen-agedcaredata . gov . au/Topics / Peopleusing-aged-care $\backslash \#$ Aged $\backslash \% 20$ care $\backslash \% 20$ use $\backslash \% 20$ and $\backslash \% 20$ age $(2021)$.

28. Australia Institute of Health and Welfare. People with disability in Australia. Australia Government. https://www . aihw.gov.au/reports/disability/ people-with-disability-in-australia / contents / social-support / younger-people-in-residential-aged-care (2020).

29. Eastwood, K. et al. Deaths of young people living in residential aged care: a national population-based descriptive epidemiological analysis of cases notified to Australian coroners. Disability and rehabilitation, 1-6 (2019).

30. Khadka, J. et al. Trends in the utilisation of aged care services in Australia, 2008-2016. BMC geriatrics 19, 1-9 (2019). 


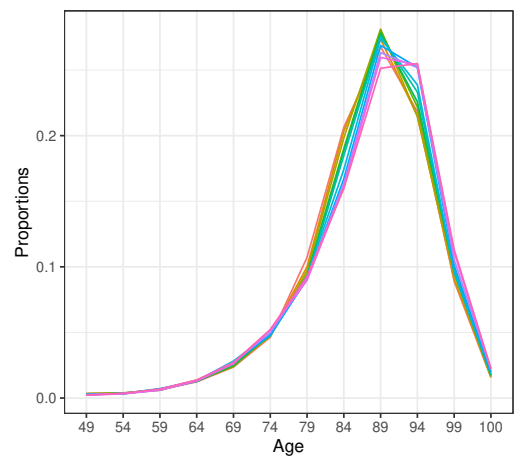

(a) Year

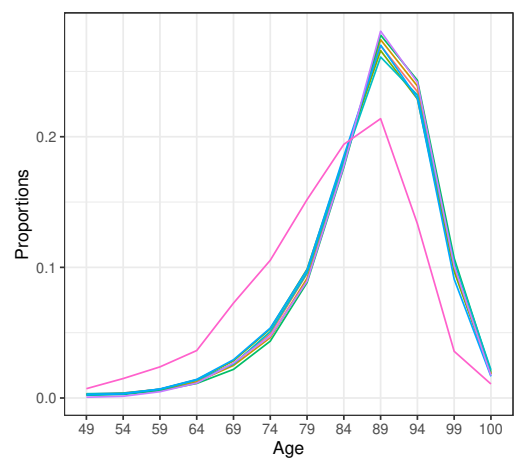

(c) State

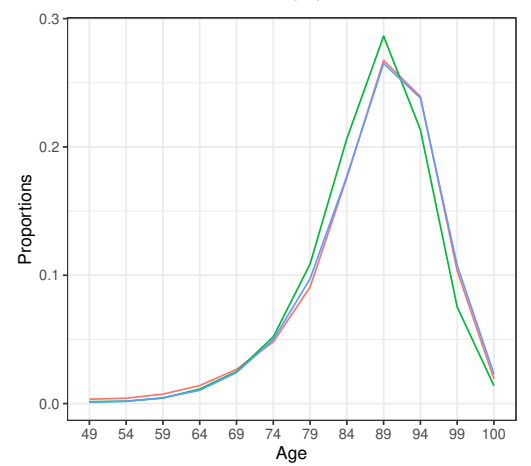

(e) Country of birth

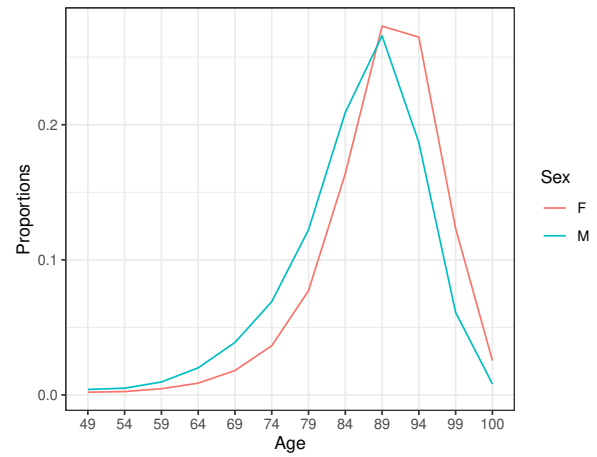

(b) Sex

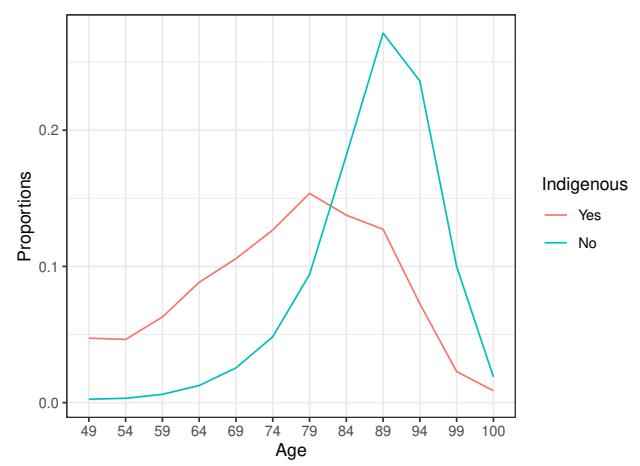

(d) Indigenous status

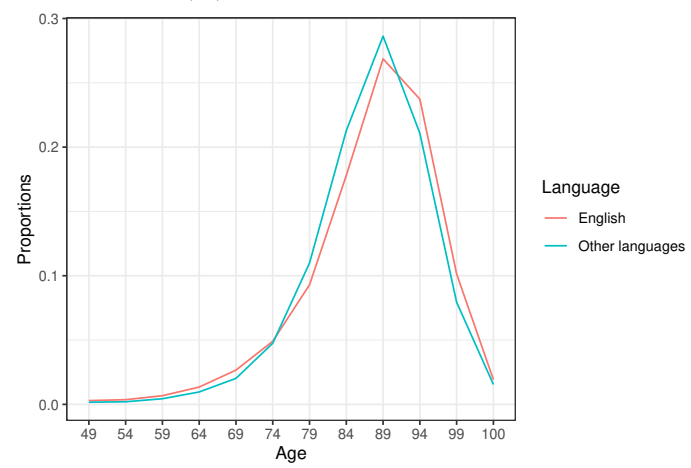

(f) Language

Figure 1: Age-specific distributions across various covariates 


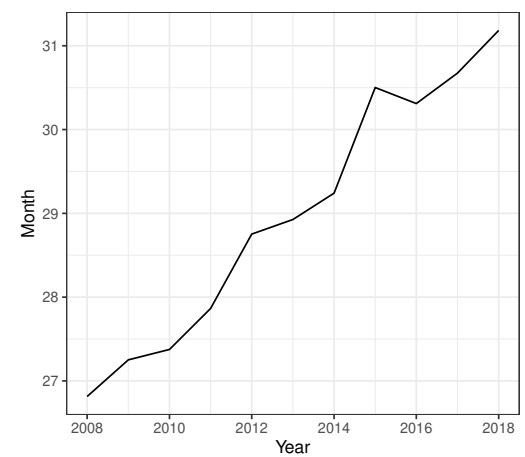

(a) Year

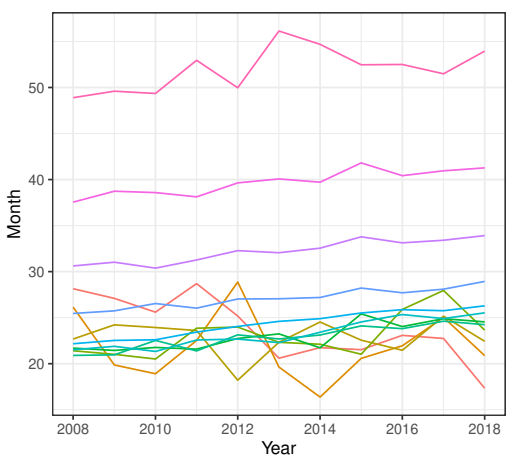

(c) Age

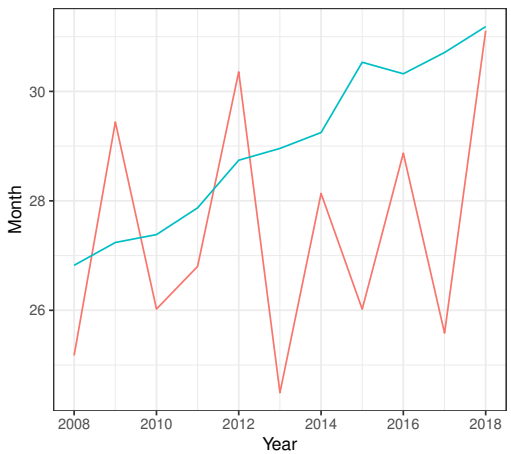

(e) Indigenous status

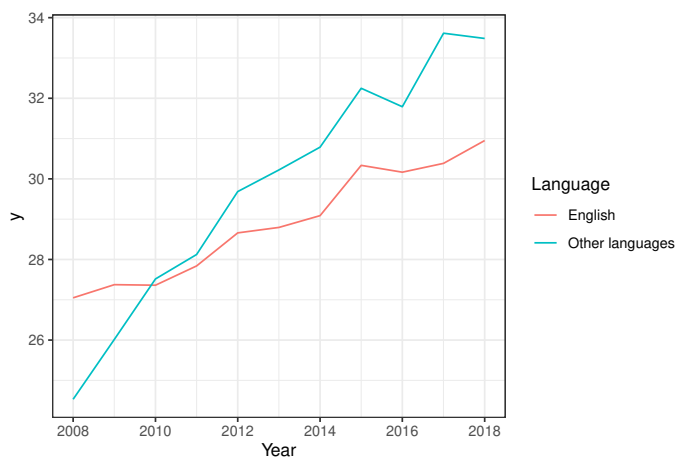

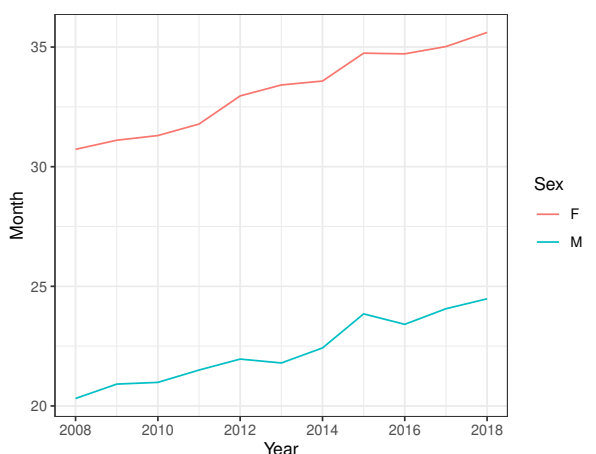

(b) Sex

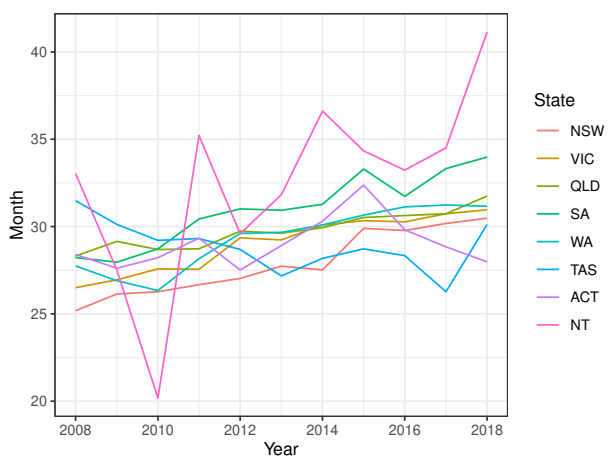

(d) State

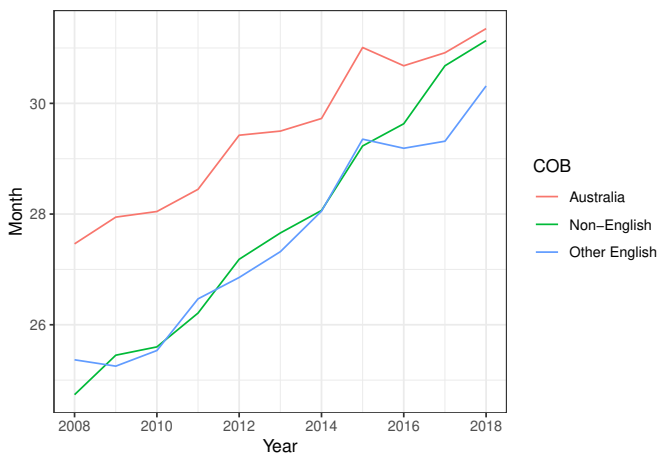

(f) Country of birth

(g) Language

Figure 2: Temporal plots of average lengths of stay at an aged care across various covariates: uncensored data over 2008-2018 


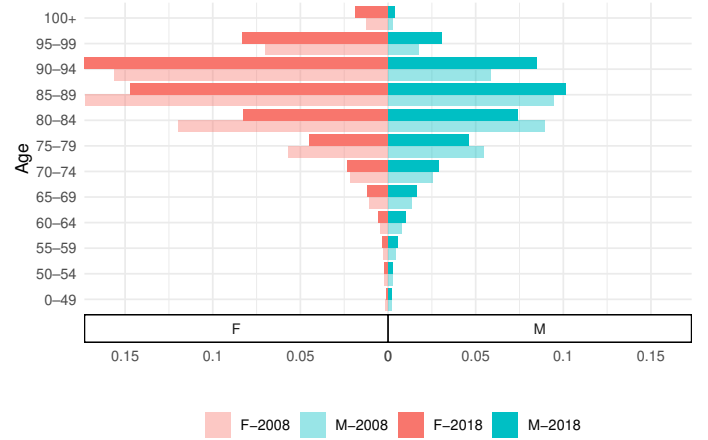

(a) New South Wales

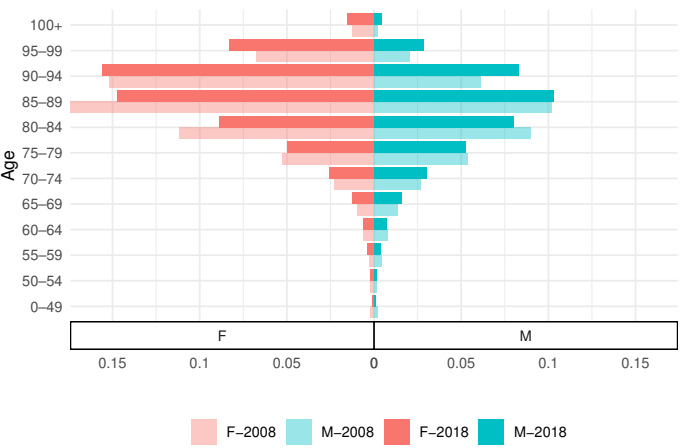

(c) Queensland

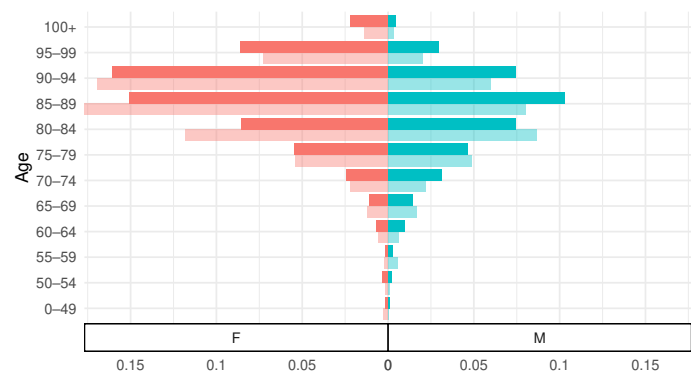

\begin{tabular}{|l|l|l|l|l|}
\hline F-2008 & M-2008 & F-2018 & M-2018
\end{tabular}

(e) Western Australia

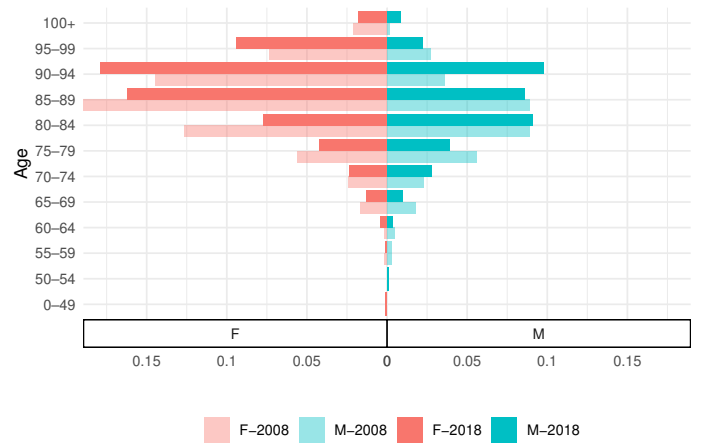

(g) Australian Capital Territory

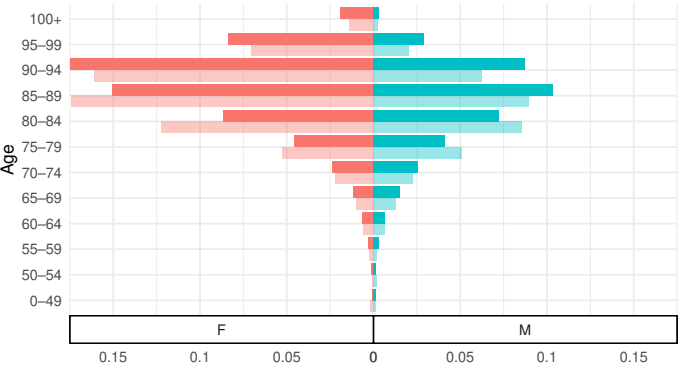

\begin{tabular}{l|l|l|l|} 
F-2008 & M-2008 & F-2018 & M-2018
\end{tabular}

(b) Victoria

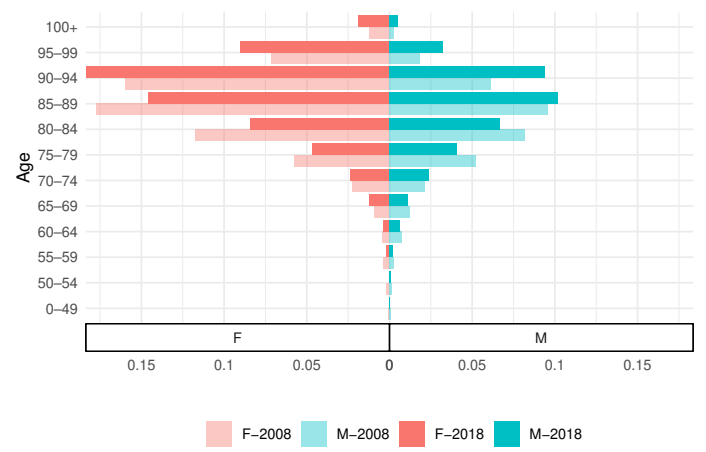

(d) South Australia

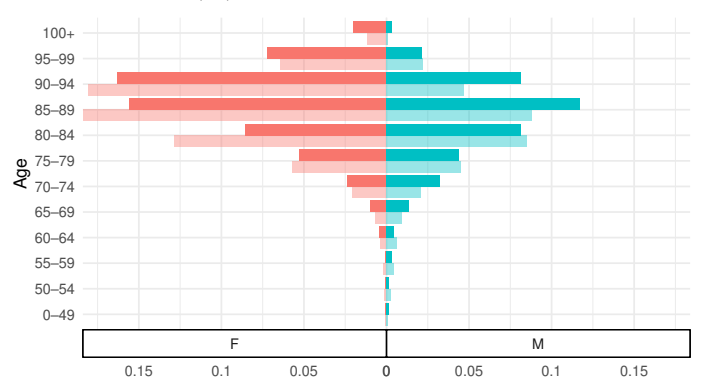

\begin{tabular}{l} 
F-2008 \\
\hline
\end{tabular}

(f) Tasmania

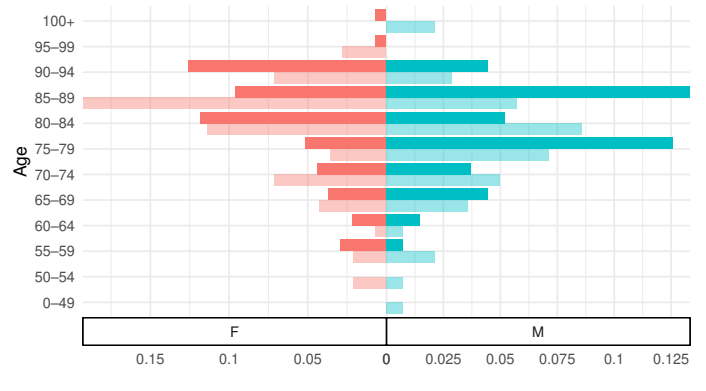

\begin{tabular}{|l|l|l|l|l|l|l|}
\hline F-2008 & M-2008 & F-2018 & M-2018
\end{tabular}

(h) Northern Territory

Figure 3: Distributions in pyramids across states 


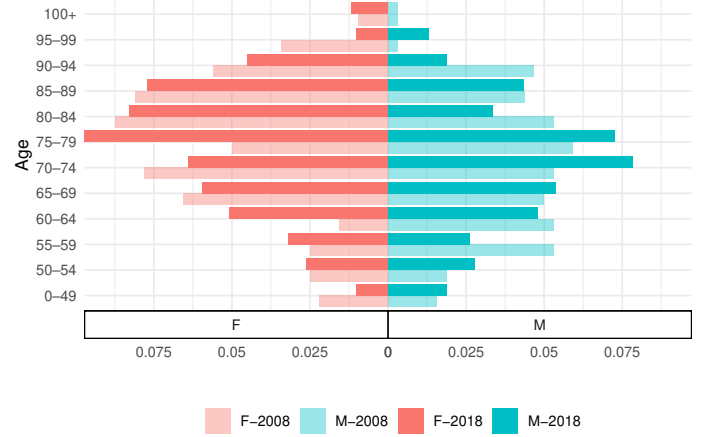

(a) Indigenous

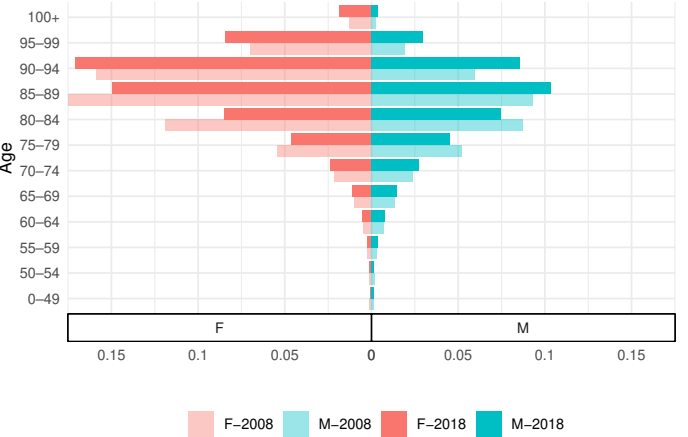

(b) Non-indigenous

Figure 4: Distributions in pyramids across indigenous status

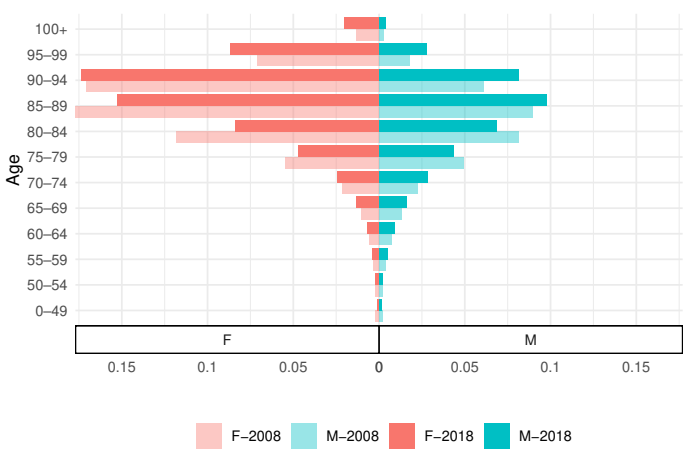

(a) Australia

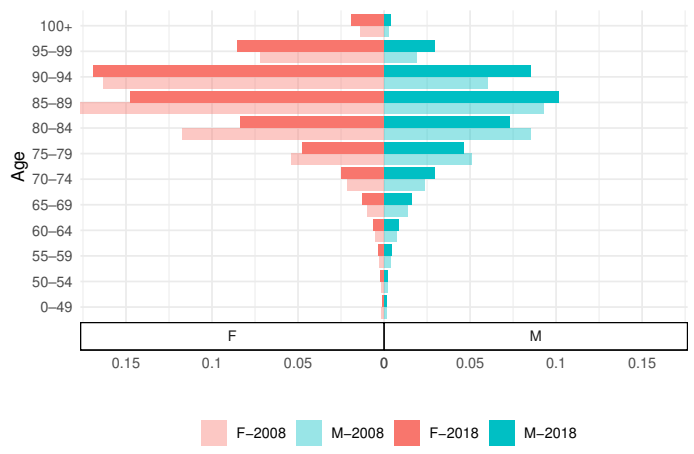

(c) Other English speaking countries

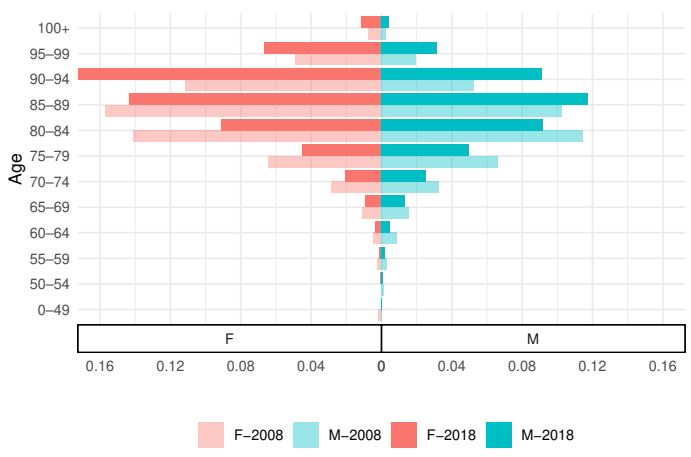

(b) Non-English speaking countries

Figure 5: Distributions in pyramids across countries of births 


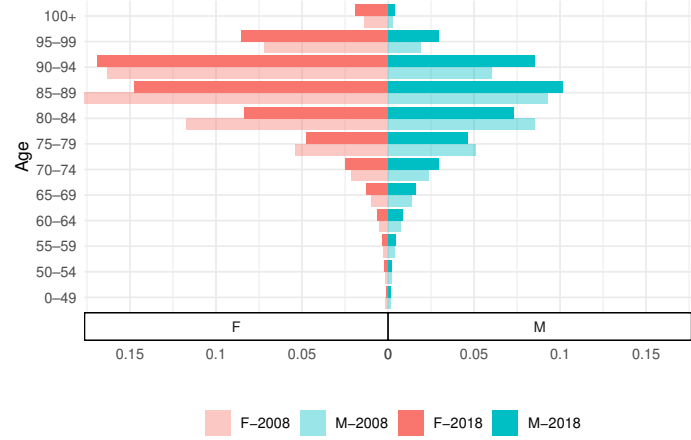

(a) English

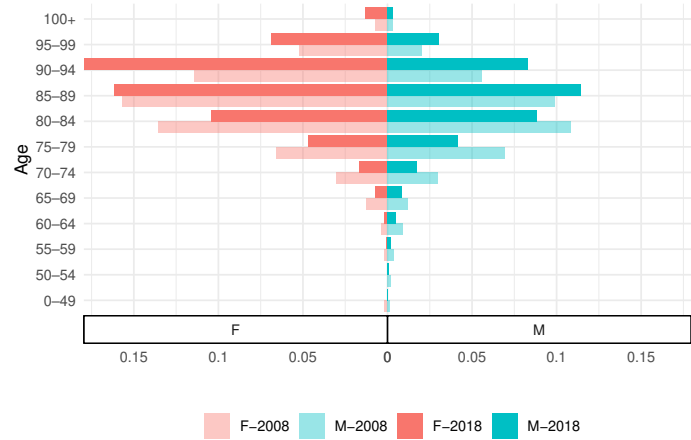

(b) Other languages

Figure 6: Distributions in pyramids across languages spoken 


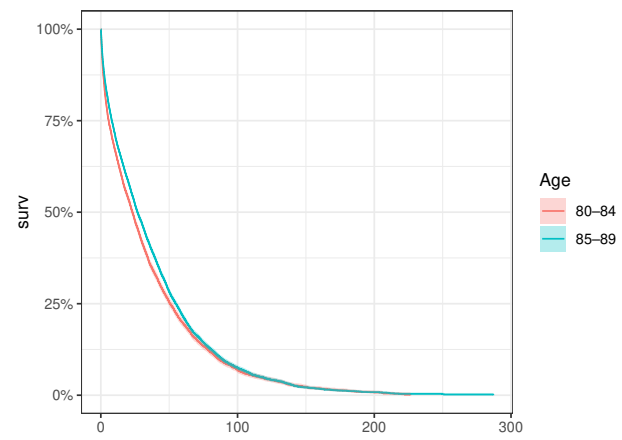

(a) Age (p-value: 0.00)

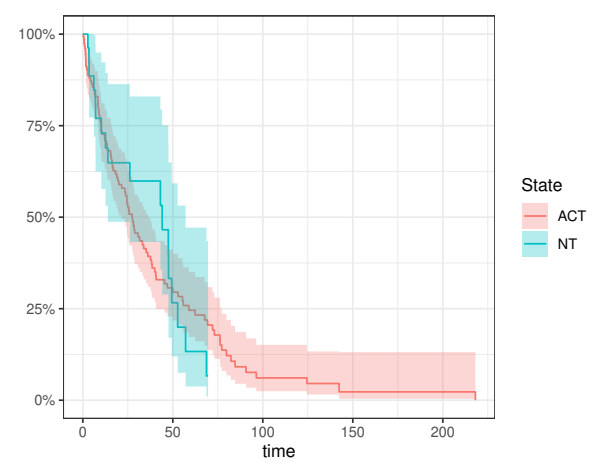

(c) State (p-value: 0.55$)$

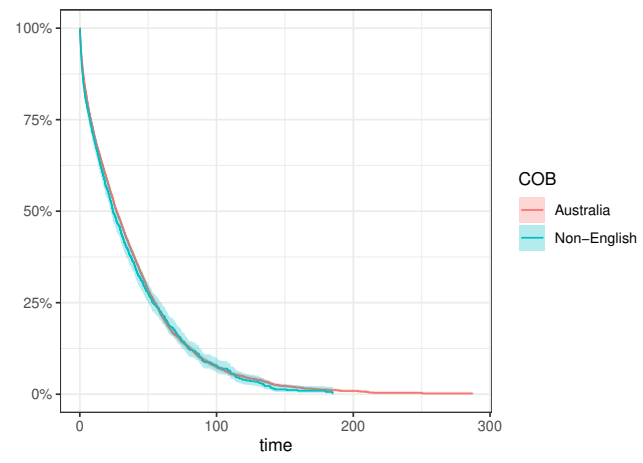

(e) Country of birth (p-value: 0.20 )

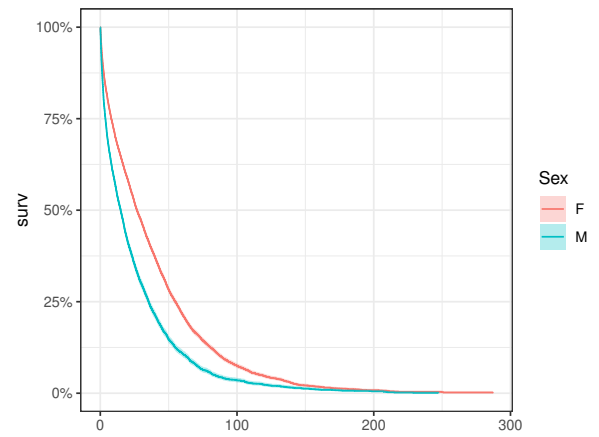

(b) Sex (p-value: 0.00)

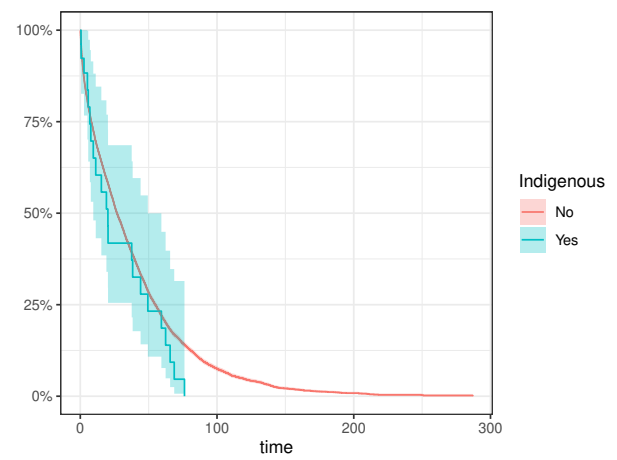

(d) Indigenous status (p-value: 0.15)

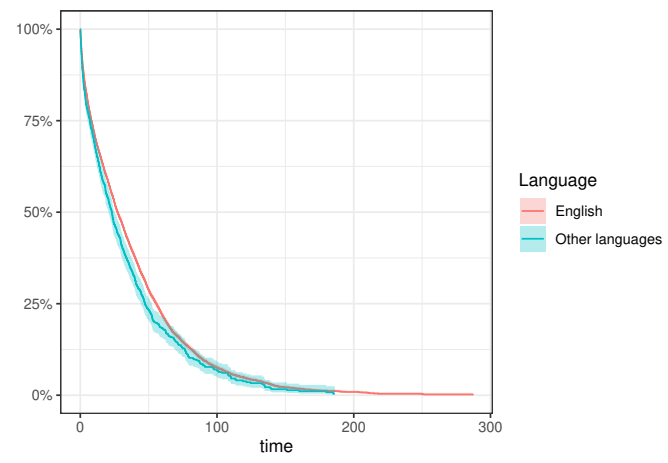

(f) Language (p-value: 0.00)

Figure 7: Preliminary analyses: Kaplan-Meier estimates 


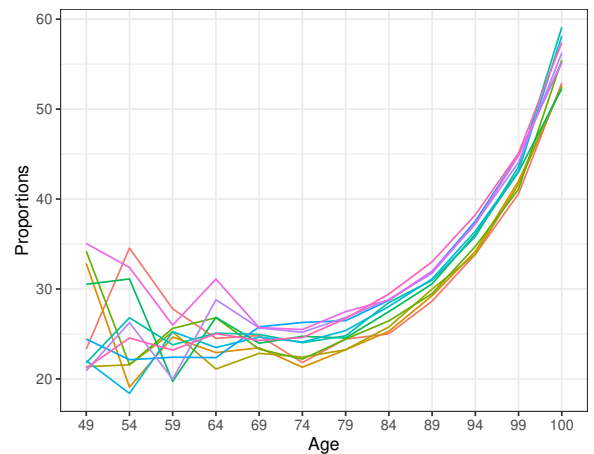

(a) Female yearly

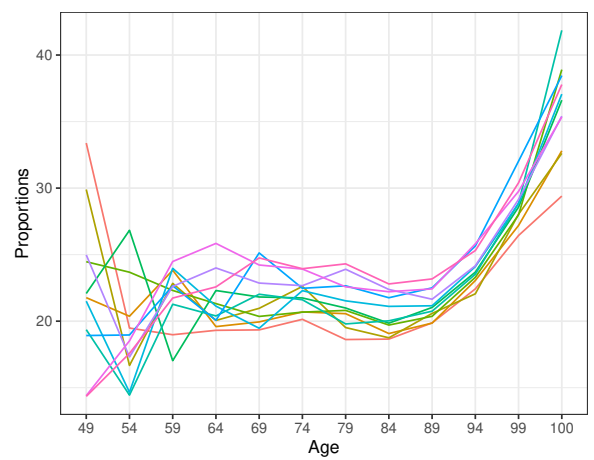

(c) Male yearly
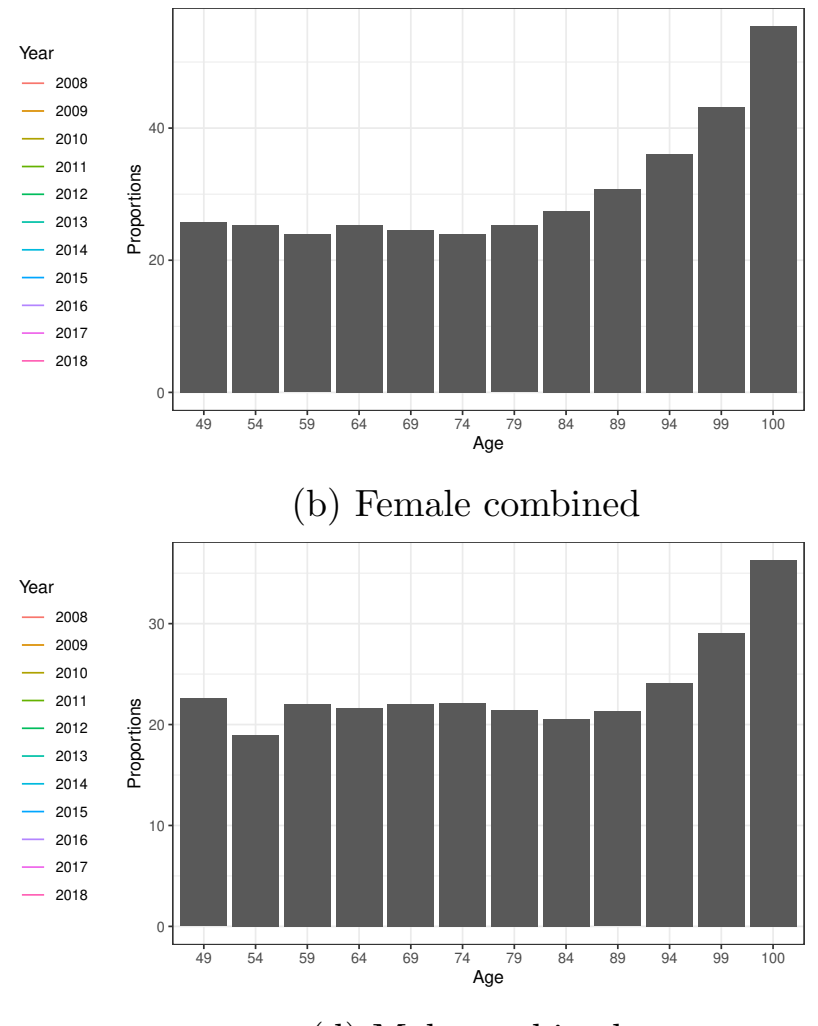

(d) Male combined

Figure 8: Preliminary analyses: Average aged care stay across original age groups

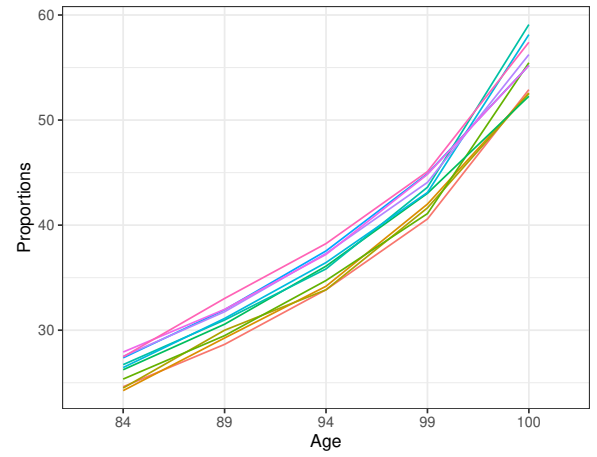

(a) Female yearly

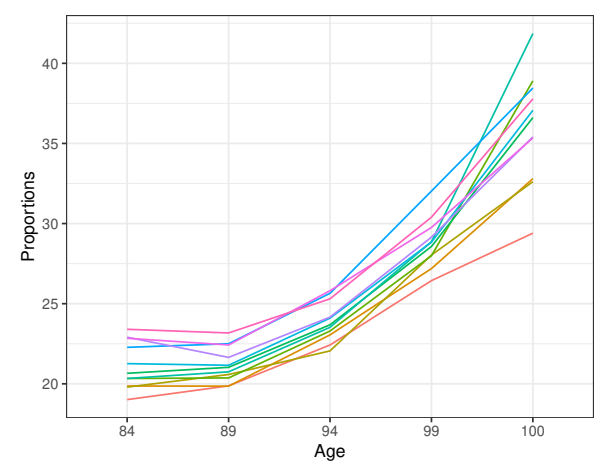

(c) Male yearly

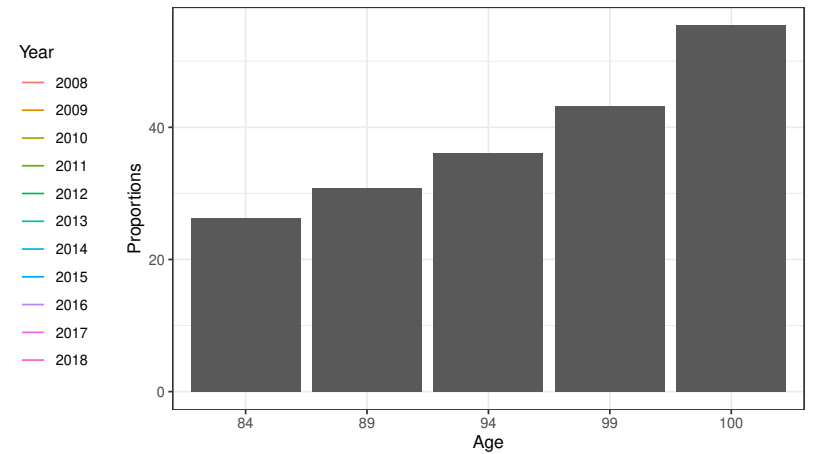

(b) Female combined
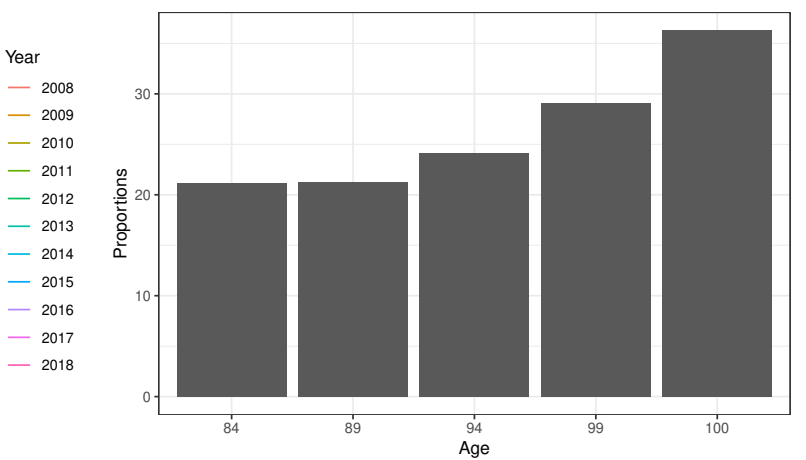

(d) Male combined

Figure 9: Preliminary analyses: Average aged care stay across combined age groups 


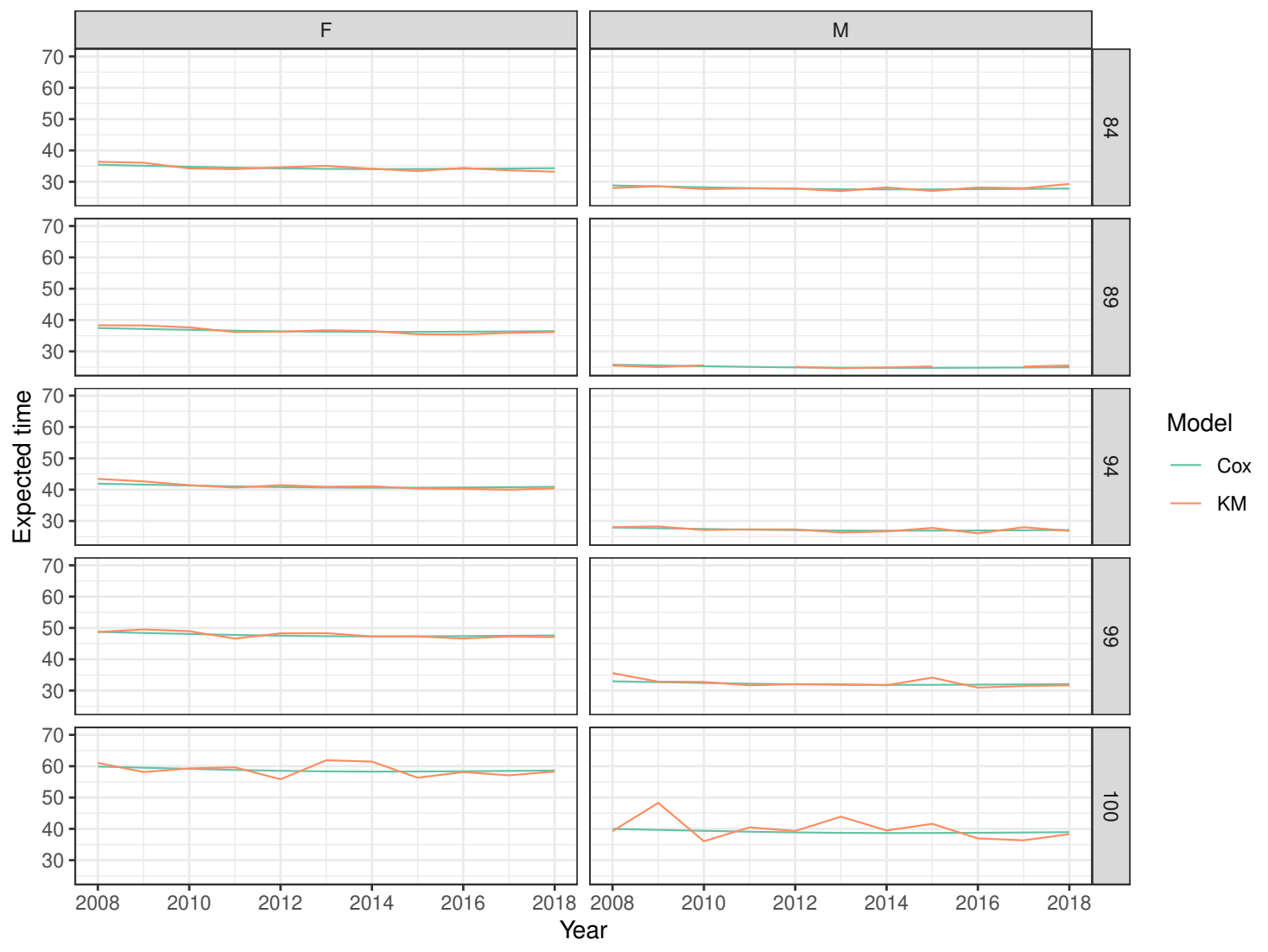

Figure 10: Fitted expected lengths of stay at an aged care: Cox regression vs Kaplan-Meier estimation 


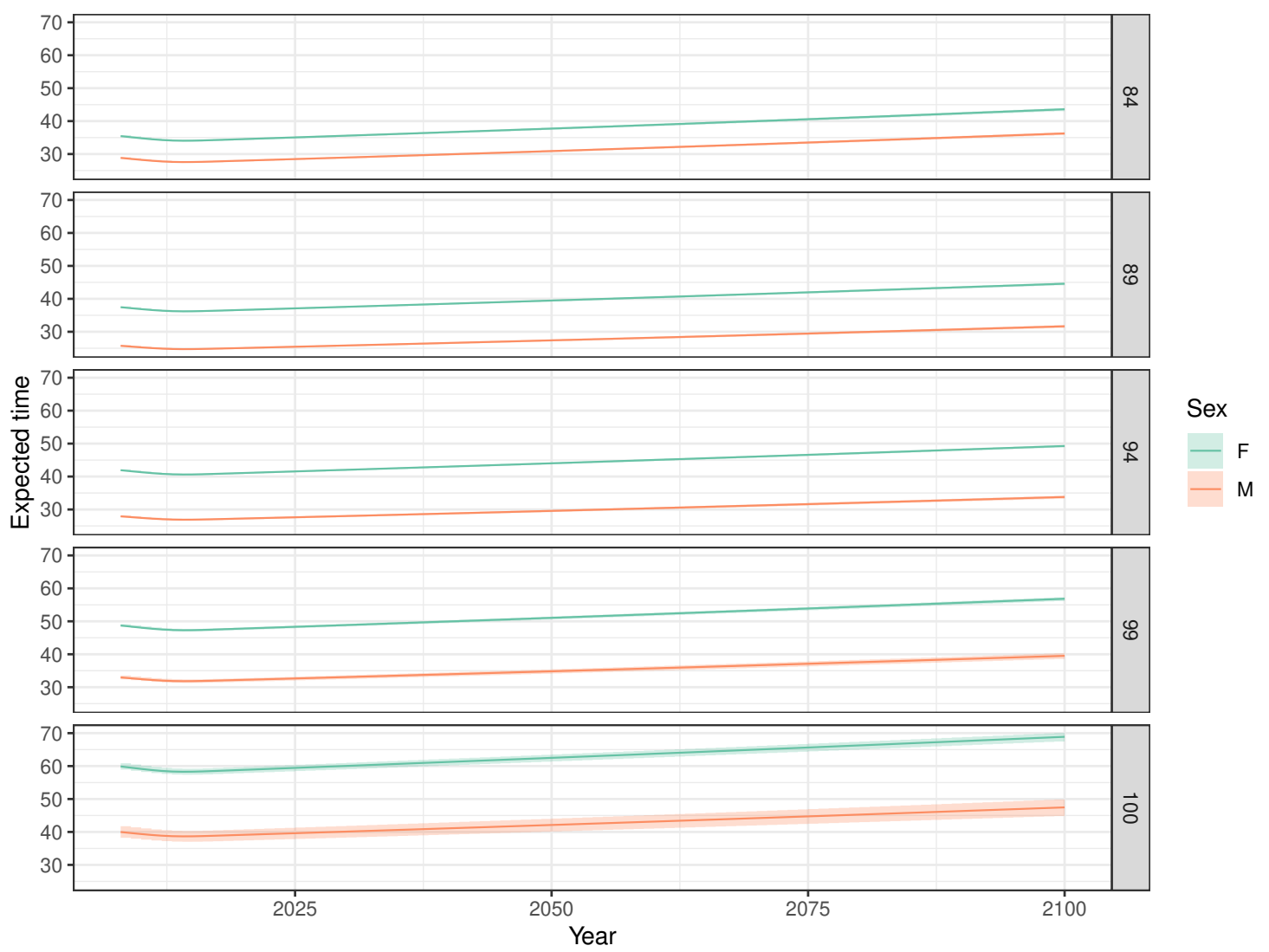

Figure 11: Fitted and predicted expected lengths of stay at an aged care: 2008-2100 


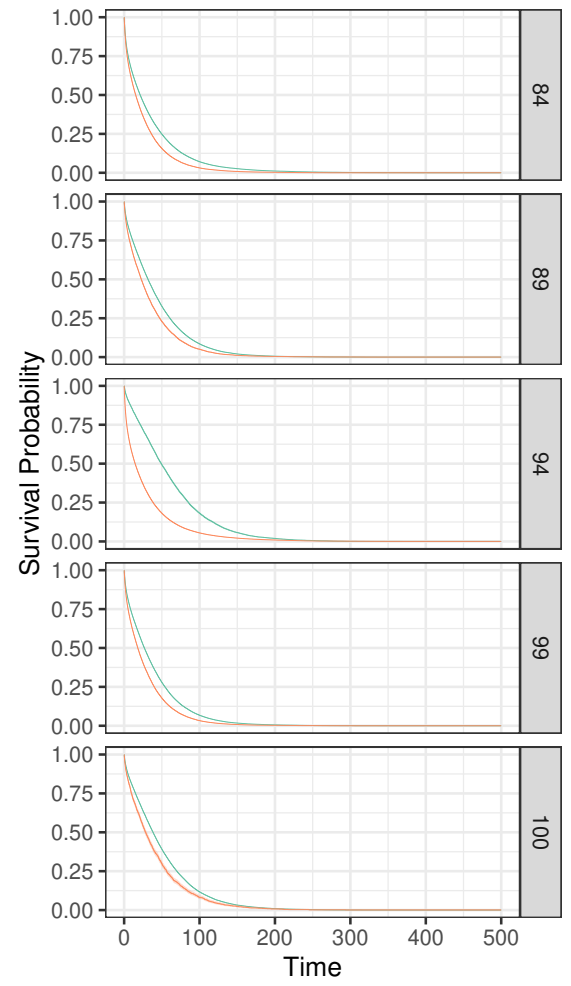

(a) 2008

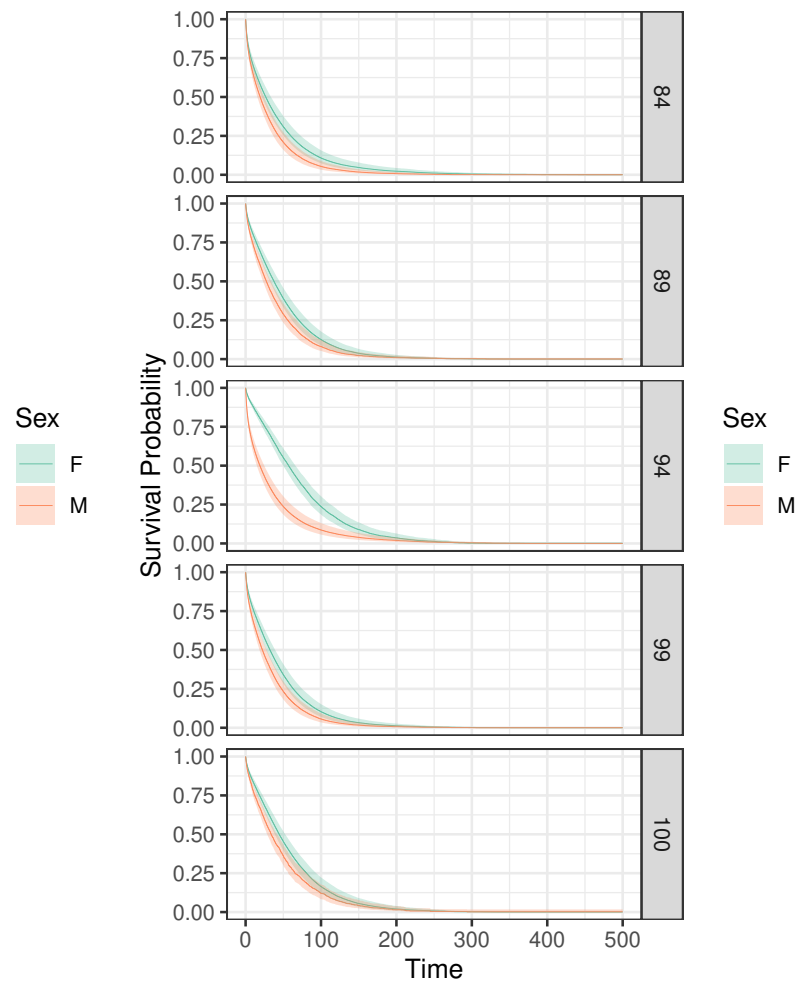

(b) 2100

Figure 12: Estimated and predicted survival curves: 2008 vs 2100 